\title{
INFINITE SUBSETS OF RANDOM SETS OF INTEGERS
}

\author{
BJøRn KJOS-HANSSEN
}

\begin{abstract}
There is an infinite subset of a Martin-Löf random set of integers that does not compute any Martin-Löf random set of integers. To prove this, we show that each real of positive effective Hausdorff dimension computes an infinite subset of a Martin-Löf random set of integers, and apply a result of Miller.
\end{abstract}

\section{Introduction}

In Reverse Mathematics [11, the Stable Ramsey's Theorem for Pairs $\left(\mathrm{SRT}_{2}^{2}\right)$ asserts for any $\Delta_{2}^{0}$ definition of a set of integers $A$ (the existence of which may not be provable in $\mathrm{SRT}_{2}^{2}$ ) the existence of an infinite subset of $A$ or of the complement of $A$. An open problem is whether $\mathrm{SRT}_{2}^{2}$ implies Weak König's Lemma (WKL) or Weak Weak König's Lemma (WWKL). WWKL asserts the existence of Martin-Löf random sets of integers. These are sets that satisfy all "computable" probability laws for the fair-coin distribution (under which each integer has probability $1 / 2$ of belonging to the set, independently of any other integer). One way to show SRT 2 implies WWKL would be to show that

(*) there is some $\Delta_{2}^{0}$ Martin-Löf random set of integers $R$ such that each infinite subset of $R$ or its complement computes a Martin-Löf random set,

and show that a proof of $(*)$ goes through in the base system for Reverse Mathematics, Recursive Comprehension $\left(\mathrm{RCA}_{0}\right)$. The simplest reason why $(*)$ would be true would be

(**) each infinite subset of any Martin-Löf random set of integers computes a Martin-Löf random set.

The statement $(* *)$ seemed fairly plausible for a while. Each Martin-Löf random set of integers $R$ is effectively immune, hence so is each infinite subset of $R$. This observation was used by Hirschfeldt et al. 4 to show that $\mathrm{SRT}_{2}^{2}$ implies the principle Diagonally Non-Recursive Functions (DNR), which asserts the existence of diagonally non-recursive functions. It is clear that WWKL should imply DNR; but the fact that WWKL is strictly stronger than DNR was only shown with considerable effort by Ambos-Spies et al. 1.

However, the assertion $(* *)$ is false, the argument having two steps. In the present paper, we show that each real of positive effective Hausdorff dimension computes an infinite subset of a Martin-Löf random set, and Miller [7 shows that there is a real of effective Hausdorff dimension 1/2 that computes no Martin-Löf random set. The truth value of $(*)$ remains unknown.

Received by the editors November 12, 2007. 
Remark 1.1. A beam splitter is a frequently used component for random number generators. Two photon detectors labeled 0 and 1 are used to detect two possible outcomes corresponding to one of two possible paths a photon can take. Thus each photon entering the beam splitter generates one random bit, 0 or 1 depending on where the photon is detected. However, photon detectors generally have an absorption efficiency that is less than $100 \%$. Can they still be used to produce a random binary sequence? Our main result can be interpreted as the anwer "no", if we merely assume that in an infinite sequence of photons, at least infinitely many of the incoming photons will be detected.

\section{Some probability theory}

The proof of our main result uses random closed sets of reals. Their study in computability theory was begun by Barmpalias et al. 2, who studied a different distribution from the one we consider here.

We sometimes consider an integer $K \in \omega=\{0,1,2, \ldots\}$ to be the set $\{0, \ldots, K-1\}$, and write $K^{<\omega}$ and $K^{\omega}$ for the sets of finite and infinite strings over $K$, respectively. For a tree $T,[T]$ denotes the closed set defined by $T$, the set of all infinite paths through $T$. If $\sigma$ is an initial substring of $\tau$ we write $\sigma \preceq \tau$. If $x \in K^{\omega}$ and $\sigma \in K^{<\omega}$ then similarly $\sigma \preceq x$ means that $\sigma$ is an initial substring of $x$. The set of all such $x$ is denoted $[\sigma]$. The concatenation of $\sigma$ and $\tau$ is denoted $\sigma * \tau$, and the length of $\sigma$ is $|\sigma|$.

Let $\mathcal{P}$ denote the power set operation. Unless otherwise stated below we have $K=2^{k}$ for some integer $k \geq 1$. $K$ plays the role of an alphabet, and a tree is a set of strings over $K$ that is closed under prefixes.

For a real number $0 \leq \ell<\infty$, let $\lambda_{k, \ell}$ be the distribution with sample space $\mathcal{P}\left(K^{<\omega}\right)$ such that each string in $K^{<\omega}$ has probability $2^{-\ell}$ of belonging to the random set, independently of any other string. We postulate no relationship between $k$ and $\ell$, but note that Theorem 3.5 below is non-vacuous only for $\ell<k$; a nice case to keep in mind is $\ell=1, k=2$.

Lemma 2.1. For all strings $\rho, \sigma, \tau$ in $K^{<\omega}$, if $\rho$ is the longest common prefix of $\sigma$ and $\tau$ with $|\sigma|=|\tau|=n$ and $|\rho|=m$, then

$$
\lambda_{k, \ell}\{S: \sigma \in S \text { and } \tau \in S\}=2^{\ell(m-2 n)} .
$$

Proof. We have

$$
\begin{gathered}
\lambda_{k, \ell}\{\sigma \in S \text { and } \tau \in S\}=\lambda_{k, \ell}\{\rho \in S\} \cdot \lambda_{k, \ell}\{\sigma \in S \text { and } \tau \in S \mid \rho \in S\} \\
=2^{-\ell m} 2^{-2 \ell(n-m)}=2^{\ell(m-2 n)} .
\end{gathered}
$$

The idea of the following Definition 2.2 is to think of a string in $K^{<\omega}$ where $K=2^{k}$ as a string in $2^{<\omega}$ of length a multiple of $k$.

Definition 2.2. Let $\iota: K \rightarrow 2^{<\omega}$ be defined by the condition that for any $a_{i} \in\{0,1\}$, $0 \leq i \leq k-1$,

$$
\iota\left(\sum_{i=0}^{k-1} a_{i} 2^{i}\right)=\left\langle a_{0}, \ldots, a_{k-1}\right\rangle
$$


For example, if $k=2$ then $\iota(3)=\langle 1,1\rangle$ and $\iota(2)=\langle 1,0\rangle$.

This is extended to a map on strings, $\iota: K^{<\omega} \rightarrow 2^{<\omega}$, by concatenation:

$$
\iota(\sigma)=\iota(\sigma(0)) * \cdots * \iota(\sigma(|\sigma|-1)) .
$$

For example, if $k=2$ then $\iota(\langle 3,2\rangle)=\langle 1,1,1,0\rangle$.

Finally $\iota$ is extended to a map on sets of strings, $\iota: \mathcal{P}\left(K^{<\omega}\right) \rightarrow \mathcal{P}\left(2^{<\omega}\right)$, by

$$
\iota(S)=\{\iota(\sigma): \sigma \in S\} .
$$

Definition $2.3\left((k, \ell)\right.$-induced distribution). For $S \subseteq K^{<\omega}, \Gamma_{S}$, the tree determined by $S$, is the (possibly empty) set of infinite paths through the $\iota$-image of the part of $S$ that is downward closed under prefixes:

$$
\Gamma_{S}=\left[\iota\left(\left\{\sigma \in K^{<\omega}: \forall \tau \preceq \sigma \tau \in S\right\}\right)\right] .
$$

The $(k, \ell)$-induced distribution $\mathbb{P}_{k, \ell}$ on the set of all closed subsets of $2^{\omega}$ is defined by

$$
\mathbb{P}_{k, \ell}(E)=\lambda_{k, \ell}\left\{S: \Gamma_{S} \in E\right\}
$$

Thus, the probability of a property $E$ of a closed subset of $2^{\omega}$ is the probability according to $\lambda_{k, \ell}$ that the $\iota$-image of a random subset of $K^{<\omega}$ determines a tree whose set of infinite paths has property $E$.

Lemma 2.4 (Chebychev-Cantelli, a special case of the Paley-Zygmund Inequality). Suppose $X$ is a nonnegative random variable, that is $X: S \rightarrow[0, \infty]$ for some sample space $S$, with probability distribution $\mathbb{P}$ on some $\sigma$-algebra $\mathcal{S} \subseteq 2^{S}$ containing the event $\{X>0\}$. Suppose $\mathbb{E}\left[X^{2}\right]<\infty$, where $\mathbb{E}$ denotes expected value.

Then

$$
\mathbb{P}\{X>0\} \geq \frac{\mathbb{E}[X]^{2}}{\mathbb{E}\left[X^{2}\right]}
$$

Proof. Since $X \geq 0$, we have $\mathbb{E}[X]=\mathbb{E}\left[X \cdot \mathbf{1}_{\{X>0\}}\right]$ where $\mathbf{1}_{E}$ is the characteristic (indicator) function of $E$ and as is customary we abbreviate $\{X: E(X)\}=\{E(X)\}=$ $E$. Squaring both sides and applying Cauchy-Schwarz yields

$$
\mathbb{E}[X]^{2}=\mathbb{E}\left[X \cdot \mathbf{1}_{\{X>0\}}\right]^{2} \leq \mathbb{E}\left[X^{2}\right] \cdot \mathbb{E}\left[\left(\mathbf{1}_{\{X>0\}}\right)^{2}\right]=\mathbb{E}\left[X^{2}\right] \cdot \mathbb{P}\{X>0\} .
$$

Let the ultrametric $v$ on $2^{\omega}$ be defined by $v(x, y)=2^{-\min \{n: x(n) \neq y(n)\}}$. For a Borel probability measure $\mu$ on $2^{\omega}$, we write $\mu(\sigma)$ for $\mu([\sigma])$.

Lemma 2.5. If $\beta>\gamma$ and $\mu$ is a Borel probability measure on $2^{\omega}$ such that for some constant $c_{R}, \mu(\sigma) \leq c_{R} 2^{-|\sigma| \beta}$ for all binary strings $\sigma$, then

$$
\iint \frac{d \mu(b) d \mu(a)}{v(a, b)^{\gamma}}<\infty
$$


Proof. Note that $\mu$ is necessarily non-atomic. Hence we have

$$
\begin{aligned}
\phi_{\gamma}(a) & :=\int \frac{d \mu(b)}{v(a, b)^{\gamma}} \leq \sum_{n=0}^{\infty} 2^{n \gamma} \mu\left\{b: v(a, b)=2^{-n}\right\}=\sum_{n=0}^{\infty} 2^{n \gamma} \mu[(a\lceil n) *(1-a(n))] \\
& \leq c_{R} \sum_{n=0}^{\infty} 2^{n \gamma} 2^{-(n+1) \beta}=c_{R} 2^{-\beta} \sum_{n=0}^{\infty} 2^{n(\gamma-\beta)}=c_{R} \frac{2^{-\beta}}{1-2^{\gamma-\beta}}=\frac{c_{R}}{2^{\beta}-2^{\gamma}} .
\end{aligned}
$$

Thus

$$
\iint \frac{d \mu(b) d \mu(a)}{v(a, b)^{\gamma}}=\int \phi_{\gamma}(a) d \mu(a) \leq \int \frac{c_{R} d \mu(a)}{2^{\beta}-2^{\gamma}}=\frac{c_{R} \mu\left(2^{\omega}\right)}{2^{\beta}-2^{\gamma}}=\frac{c_{R}}{2^{\beta}-2^{\gamma}}<\infty .
$$

Lemma 2.6. Suppose we are given reals $\beta>\gamma=\frac{\ell}{k}$. Let $\mu$ be any Borel probability measure on $2^{\omega}$ such that

$$
\iint \frac{d \mu(b) d \mu(a)}{v(a, b)^{\gamma}}=c<\infty
$$

Let $\Gamma$ be the (closed set valued) random variable whose value is the outcome of an experiment obeying distribution $\mathbb{P}_{k, \ell}$, the $(k, \ell)$-induced distribution. For any closed set $A$, we have

$$
\mathbb{P}_{k, \ell}\{\Gamma: \Gamma \cap A \neq \varnothing\} \geq \frac{\mu(A)^{2}}{c} .
$$

Proof. Let $\mathfrak{C}_{n}=\{0,1\}^{n}$, the set of all binary strings of length $n$. Let $S$ denote the random variable that is the outcome of the experiment according to the distribution $\lambda_{k, \ell} ;$ so $S$ takes values in $\mathcal{P}\left(K^{<\omega}\right)$. Let $n$ be a positive integer that is a multiple of $k$, let $\mathfrak{S}_{n}=\left\{\sigma:|\sigma|=n\right.$ and $\left.\iota^{-1}(\sigma) \in S\right\}$, and

$$
Y_{n}=\sum_{\sigma \in \mathfrak{S}_{n},[\sigma] \cap A \neq \varnothing} \frac{\mu(\sigma)}{2^{-|\sigma| \gamma}}=\sum_{\sigma \in \mathfrak{C}_{n},[\sigma] \cap A \neq \varnothing} \mu(\sigma) 2^{n \gamma} \mathbf{1}_{\left\{\sigma \in \mathfrak{S}_{n}\right\}}
$$

Let us write $\mathbb{P}$ for $\lambda_{k, \ell}$. Note that $\mathbb{E}\left[\mathbf{1}_{\left\{\sigma \in \mathfrak{S}_{n}\right\}}\right]=\mathbb{P}\left\{\sigma \in \mathfrak{S}_{n}\right\}=\left(2^{-\ell}\right)^{n / k}=2^{-n \gamma}$.

Thus by linearity of expectation $\mathbb{E}$,

$$
\mathbb{E}\left[Y_{n}\right]=\sum_{\sigma \in \mathfrak{C}_{n},[\sigma] \cap A \neq \varnothing} \mu(\sigma) 2^{n \gamma} \mathbb{E}\left[\mathbf{1}_{\left\{\sigma \in \mathfrak{S}_{n}\right\}}\right]=\sum_{\sigma \in \mathfrak{C}_{n},[\sigma] \cap A \neq \varnothing} \mu(\sigma) \geq \mu(A) .
$$

Next,

$$
\mathbb{E}\left[Y_{n}^{2}\right]=\sum_{\sigma \in \mathfrak{C}_{n},[\sigma] \cap A \neq \varnothing} \sum_{\tau \in \mathfrak{C}_{n},[\tau] \cap A \neq \varnothing} \mu(\sigma) \mu(\tau) 2^{2 n \gamma} \mathbb{P}\left\{\sigma \in \mathfrak{S}_{n} \text { and } \tau \in \mathfrak{S}_{n}\right\},
$$

because $\mathbb{E}\left[\mathbf{1}_{\left\{\sigma \in \mathfrak{S}_{n}\right\}} \mathbf{1}_{\left\{\tau \in \mathfrak{S}_{n}\right\}}\right]=\mathbb{P}\left\{\sigma \in \mathfrak{S}_{n}\right.$ and $\left.\tau \in \mathfrak{S}_{n}\right\}$.

Let $\sigma$ and $\tau$ be binary strings, let $m_{\sigma, \tau}$ be the length of the common prefix of $\sigma$ and $\tau$, and let $m_{\sigma, \tau}^{\prime}=\max \left\{k q: k q \leq m_{\sigma, \tau}, q \in \omega\right\}$, which is the length of $\iota$ of the common prefix of $\iota^{-1}(\sigma)$ and $\iota^{-1}(\tau)$. Let $\widehat{n}=n / k$, and $\widehat{m}_{\sigma, \tau}=m_{\sigma, \tau}^{\prime} / k$, which is the length of the common prefix of $\iota^{-1}(\sigma)$ and $\iota^{-1}(\tau)$.

Note that $\iota^{-1}(\sigma)$ is a $K$-ary string of length $n / k$. By Lemma 2.1.

$$
\begin{gathered}
\mathbb{P}\left\{\sigma \in \mathfrak{S}_{n} \text { and } \tau \in \mathfrak{S}_{n}\right\}=\lambda_{k, \ell}\left(\left\{S: \iota^{-1}(\sigma) \in S \text { and } \iota^{-1}(\tau) \in S\right\}\right) \\
\leq 2^{\ell\left(\widehat{m}_{\sigma, \tau}-2 \widehat{n}\right)}=2^{\gamma\left(m_{\sigma, \tau}^{\prime}-2 n\right)} \leq 2^{\gamma\left(m_{\sigma, \tau}-2 n\right)} .
\end{gathered}
$$


Note that if $x \in[\sigma]$ and $y \in[\tau]$ then $v(x, y) \leq 2^{-m_{\sigma, \tau}}$. Therefore

$$
\begin{aligned}
& \mathbb{E}\left[Y_{n}^{2}\right] \leq \sum_{\sigma \in \mathfrak{C}_{n},[\sigma] \cap A \neq \varnothing} \sum_{\tau \in \mathfrak{C}_{n},[\sigma] \cap A \neq \varnothing} \mu(\sigma) \mu(\tau) 2^{\gamma m_{\sigma, \tau}} \\
& \leq \sum_{\sigma \in \mathfrak{C}_{n}} \sum_{\tau \in \mathfrak{C}_{n}} \mu(\sigma) \mu(\tau) 2^{\gamma m_{\sigma, \tau}} \leq \iint \frac{d \mu(x) d \mu(y)}{v(x, y)^{\gamma}}=c .
\end{aligned}
$$

By Lemma 2.4,

$$
\mathbb{P}\left\{Y_{n}>0\right\} \geq \frac{\mathbb{E}\left[Y_{n}\right]^{2}}{\mathbb{E}\left[Y_{n}^{2}\right]} \geq \frac{\mu(A)^{2}}{c}
$$

Since $A$ is closed, and since $Y_{n+k}>0$ implies $Y_{n}>0$, we can conclude (letting $n$ still range over multiples of $k$ )

$$
\mathbb{P}_{k, \ell}\{A \cap \Gamma \neq \varnothing\} \geq \mathbb{P}\left\{Y_{n}>0 \text { for all } n\right\}=\lim _{n \rightarrow \infty} \mathbb{P}\left\{Y_{n}>0\right\} \geq \frac{\mu(A)^{2}}{c}
$$

A result similar to Lemma 2.6 but for percolation limit sets rather than for the $(k, \ell)$-induced distribution, was obtained by Lyons [6] building on work of Hawkes [3].

\section{Martin-Löf random sets}

For a real number $0 \leq \gamma \leq 1$, the $\gamma$-weight wt $_{\gamma}(C)$ of a set of strings $C$ is defined by

$$
\mathrm{wt}_{\gamma}(C)=\sum_{w \in C} 2^{-|w| \gamma}
$$

A Martin-Löf $\gamma$-test is a uniformly computably enumerable (c.e.) sequence $\left(U_{n}\right)_{n<\omega}$ of sets of strings such that

$$
(\forall n)\left(\mathrm{wt}_{\gamma}\left(U_{n}\right) \leq 2^{-n}\right) .
$$

For a set of strings $V$, let $[V] \preceq=\bigcup\{[\sigma]: \sigma \in V\}$ be the open subset of $2^{\omega}$ defined by $V$. A real is $\gamma$-random if it does not belong to $\cap_{n}\left[U_{n}\right] \preceq$ for any $\gamma$-test $\left(U_{n}\right)_{n<\omega}$. If $\gamma=1$ we simply say that the real, or the set of integers $\{n: x(n)=1\}$, is Martin-Löf random.

For a (Borel) probability measure $\mu$ and a real $x$, we say that $x$ is $\mu$-random if for each sequence $\left(U_{n}\right)_{n<\omega}$ that is uniformly c.e. in $\mu$ and where $\mu\left[U_{n}\right]^{\preceq} \leq 2^{-n}$ for all $n$, we have $x \notin \cap_{n}\left[U_{n}\right] \preceq$. (Note that $\mu$ can be considered as an oracle via an encoding of the reals $\mu([\sigma]), \sigma \in 2^{<\omega}$; this definition is due to Reimann and Slaman.)

We say $x$ is $\gamma$-capacitable if $x$ is $\mu$-random with respect to some probability measure $\mu$ such that for some $c$,

$$
\forall \sigma\left[\mu(\sigma) \leq c 2^{-\gamma|\sigma|}\right]
$$

$x$ is $\gamma$-energy random if $x$ is $\mu$-random with respect to some probability measure $\mu$ such that

$$
\iint \frac{d \mu(b) d \mu(a)}{v(a, b)^{\gamma}}<\infty .
$$

If we only require that $x \notin \bigcap_{n}\left[U_{n}\right]^{\preceq}$ when $\left(U_{n}\right)_{n<\omega}$ is c.e., as opposed to c.e. relative to $\mu$, then we say that $x$ is Hippocrates $\mu$-random, $\gamma$-capacitable, or $\gamma$-energy random, respectively. As the reader may recall, Hippocrates did not consult the oracle of 
Delphi, and similarly a test for Hippocrates randomness $U_{n}$ does not consult the oracle $\mu$.

Effective Hausdorff dimension was introduced by Lutz [5] and is a notion of partial randomness. For example, if the sequence $x_{0} x_{1} x_{2} \cdots$ is Martin-Löf random, then the sequence $x_{0} 0 x_{1} 0 x_{2} 0 \cdots$ has effective Hausdorff dimension equal to $\frac{1}{2}$. Let $\operatorname{dim}_{H}^{1} x$ denote the effective (or constructive) Hausdorff dimension of $x$; then we have $\operatorname{dim}_{H}^{1}(x)=\sup \{\gamma: x$ is $\gamma$-random $\}$ (Reimann and Stephan [10]).

Theorem 3.1 (Reimann [9]). For any real $x \in 2^{\omega}$,

$$
\operatorname{dim}_{H}^{1} x=\sup \{\beta: x \text { is } \beta \text {-capacitable }\} \text {, }
$$

where $\sup \varnothing=0$.

Lemma 3.2. Suppose $U$ is a c.e. set of strings with effective enumeration $U=$ $\bigcup_{s<\omega} U_{s}$. Then

is a $\Sigma_{1}^{0}$ class.

$$
\left\{y \in 2^{\omega}:\left\{S: y \in \Gamma_{S}\right\} \subseteq[U]^{\preceq}\right\}
$$

Proof. For a closed set $\Gamma$, let us write $\Gamma \uparrow m$ for $\bigcup\{[\sigma]:|\sigma|=m,[\sigma] \cap \Gamma \neq \varnothing\}$. As is usual, for an oracle $A$, a Turing reduction $\{e\}$ and a stage $s$, let $\{e\}_{s}^{A}(n)$ denote the output if any of the computation on input $n$ by stage $s$, and $\{e\}^{A}(n)$ the value by the time the computation halts, if ever. For some $e$, we have

$$
\begin{gathered}
\left\{y \in 2^{\omega}:\left\{S: y \in \Gamma_{S}\right\} \subseteq[U] \preceq\right\}=\left\{y: \forall S\left(y \in \Gamma_{S} \rightarrow S \in[U] \preceq\right)\right\} \\
=\left\{y: \forall S\left(\forall m\left(y\left\lceil m \in \Gamma_{S}\lceil m) \rightarrow S \in[U] \preceq\right)\right\}\right.\right. \\
=\left\{y: \forall S \exists m, s\left(y\left\lceil m \notin \Gamma_{S}\left\lceil m \text { or } S \in\left[U_{s}\right]^{\preceq}\right)\right\}\right.\right. \\
=\left\{y: \forall S\left(\{e\}^{S \oplus y}(0) \downarrow\right)\right\}=\left\{y: \exists s \forall \sigma \in 2^{s}\left(\{e\}_{s}^{\sigma \oplus y\lceil s}(0) \downarrow\right)\right\} .
\end{gathered}
$$

Definition 3.3. Let $f_{k}: \omega \rightarrow K^{<\omega}$ be an effective bijection. A closed set is called Martin-Löf random according to the $(k, \ell)$-induced distribution if it is of the form $\Gamma_{S}$ for some set $S$ such that $f_{k}^{-1}(S)$ is a Martin-Löf random subset of $\omega$ with respect to Bernoulli measure with parameter $2^{-\ell}$.

For $\ell=1$, Definition 3.3 states that $f_{k}^{-1}(S)$ is a Martin-Löf random subset of $\omega$ as defined above. Although we will not need it, one can show that Definition 3.3 is independent of the choice of $f_{k}$.

Lemma 3.4. Let $\mu$ be any Borel probability measure on $2^{\omega}$. Then for each open set $U$, there is a clopen set $A \subseteq U$ with $\mu(A) \geq \mu(U)-\epsilon$.

Proof. Write $U=\bigcup_{n} D_{n}$ where the $D_{n}$ are clopen and disjoint. These $D_{n}$ exist by topological properties of $2^{\omega}$. Then $\infty>1 \geq \mu U=\mu \bigcup_{n} D_{n}=\sum_{n} \mu\left(D_{n}\right)$. Choose $n$ such that $\sum_{k>n} \mu\left(D_{k}\right) \leq \epsilon$ and let $A=D_{0} \cup \cdots \cup D_{n}$. Then $\mu A=$ $\mu(U)-\sum_{k>n} \mu\left(D_{k}\right) \geq \mu(U)-\epsilon$.

Theorem 3.5. Let $\gamma=\frac{\ell}{k}$. Each Hippocrates $\gamma$-energy random real belongs to a Martin-Löf random closed set under the $(k, \ell)$-induced distribution. 
Proof. Let $x$ be any real and suppose $x$ belongs to no Martin-Löf random closed set according to $\mathbb{P}_{k, \ell}$. That is, $\left\{S: x \in \Gamma_{S}\right\}$ contains no set $S$ that is Martin-Löf random according to the $(k, \ell)$-distribution. Thus if we let $V_{n}$ be a universal Martin-Löf test for $\lambda_{k, \ell}$ then $\left\{S: x \in \Gamma_{S}\right\} \subseteq \cap_{n}\left[V_{n}\right]^{\preceq}$. So $x \in \cap_{n<\omega}\left[U_{n}\right]^{\preceq}$, where

$$
\left[U_{n}\right]^{\preceq}=\left\{y \in 2^{\omega}:\left\{S: y \in \Gamma_{S}\right\} \subseteq\left[V_{n}\right]^{\preceq}\right\} .
$$

We have already seen in Lemma 3.2 that $\left[U_{n}\right]^{\preceq}$ is $\Sigma_{1}^{0}$, and by the proof $\left\{\left[U_{n}\right]^{\preceq}\right.$ : $n<\omega\}$ is even $\Sigma_{1}^{0}$ uniformly in $n$. Thus we may choose $U_{n}, n<\omega$ as uniformly c.e. sets of strings. To show $x$ is not Hippocrates $\gamma$-energy random, we must show that for any $\mu$ with $\iint \frac{d \mu(a) d \mu(b)}{v(a, b)^{\gamma}}<\infty, x$ is not Hippocrates $\mu$-random. We do this by showing that some effectively given subsequence of $\left\{U_{n}\right\}_{n<\omega}$ is a $\mu$-Martin-Löf test.

By Lemma 3.4 for each $\epsilon>0$ there is a clopen set $A \subseteq\left[U_{n}\right] \preceq$ with $\mu A \geq \mu\left[U_{n}\right] \preceq-\epsilon$.

Since

$$
\left\{S \subseteq K^{<\omega}: \Gamma_{S} \cap\left[U_{n}\right]^{\preceq} \neq \varnothing\right\}=\bigcup_{y \in\left[U_{n}\right] \preceq}\left\{S: y \in \Gamma_{S}\right\} \subseteq\left[V_{n}\right]^{\preceq},
$$

it follows from Lemma 2.6 that

$$
\frac{\mu(A)^{2}}{c} \leq \lambda_{k, \ell}\left\{S: \Gamma_{S} \cap A \neq \varnothing\right\} \leq \lambda_{k, \ell}\left[V_{n}\right]^{\prec} \leq 2^{-n}
$$

so $\mu\left(\left[U_{n}\right]^{\preceq}\right) \leq \mu(A)+\varepsilon \leq \sqrt{c 2^{-n}}+\varepsilon$. Since $\varepsilon>0$ was arbitrary, $\mu\left(\left[U_{n}\right]^{\preceq}\right) \leq \sqrt{c 2^{-n}}$. After taking an effective subsequence we can replace $\sqrt{c 2^{-n}}$ by $2^{-n}$, and so we are done.

Theorem 3.6. Each member of any Martin-Löf random closed set under the $(k, \ell)$ induced distribution is truth-table equivalent to an infinite subset of a Martin-Löf random set under $\lambda_{k, \ell}$.

Proof. Let $x \in \Gamma_{S}$ where $S$ is Martin-Löf random under $\lambda_{k}$. Let $Y=\{\sigma \in S: \iota(\sigma) \preceq$ $x\}$ where as above $\sigma \preceq x$ denotes that $\sigma$ is an initial segment of $x$. Since $x \in \Gamma_{S}$, $Y$ is infinite. Since $\tau \preceq x \Leftrightarrow \iota^{-1}(\tau) \in Y$ for $\tau$ of length a multiple of $k$, and since $\sigma \in Y \Leftrightarrow \iota(\sigma) \preceq x, x$ is truth-table equivalent to $Y$.

Theorem 3.7. Each real of positive effective Hausdorff dimension computes (and in fact is truth-table equivalent to) an infinite subset of a Martin-Löf random set of integers.

Proof. Let $x$ be a real of positive effective Hausdorff dimension, let $\ell=1$ and let $k$ be such that $\operatorname{dim}_{H}^{1}(x)>\gamma:=\frac{\ell}{k}=\frac{1}{k}$. By Theorem 3.1. $x$ is $\beta$-capacitable for some $\beta>\gamma$. By Lemma 2.5, $x$ is $\gamma$-energy random and hence Hippocrates $\gamma$-energy random. By Theorem 3.5, $x$ is a member of a Martin-Löf random closed set under the $(k, \ell)$-induced distribution. By Theorem 3.6, $x$ is Turing equivalent to an infinite subset $Y$ of a Martin-Löf random set under $\lambda_{k, \ell}$. Now $\lambda_{k, \ell}$ is the uniform distribution on subsets of $K^{<\omega}$ with success probability $2^{-\ell}=\frac{1}{2}$. Thus $f_{k}^{-1}(Y)$ is an infinite subset of a Martin-Löf random set of integers, and truth-table equivalent to $Y$. 
Theorem 3.8. There is an infinite subset of a Martin-Löf random set of integers that computes no Martin-Löf random set of integers.

Proof. Miller [7] shows that there is a real $x$ of effective Hausdorff dimension $1 / 2$ which does not compute any Martin-Löf random real. By Theorem 3.7, $x$ computes an infinite subset of a Martin-Löf random set.

We only needed the case $\ell=1$ for Theorem 3.8 . If we let $\ell \rightarrow \infty$, we see that even a Martin-Löf random set of integers with respect to an arbitrarily small probability of membership $2^{-\ell}$ may have an infinite subset that computes no Martin-Löf random set.

\section{Acknowledgments}

The author was partially supported by NSF grant DMS-0652669, and thanks Soumik Pal for pointing out the existence of the work of Hawkes and Lyons [3] and its exposition by Mörters and Peres [8].

\section{References}

[1] K. Ambos-Spies, B. Kjos-Hanssen, S. Lempp, and T. A. Slaman, Comparing DNR and WWKL, J. Symbolic Logic 69 (2004), no. 4, 1089-1104.

[2] G. Barmpalias, P. Brodhead, D. Cenzer, S. Dashti, and R. Weber, Algorithmic randomness of closed sets, J. Logic Comput. 17 (2007), no. 6, 1041-1062.

[3] J. Hawkes, Trees generated by a simple branching process, J. London Math. Soc. (2) 24 (1981), no. $2,373-384$.

[4] D. R. Hirschfeldt, C. G. Jockusch, B. Kjos-Hanssen, S. Lempp, and T. A. Slaman, The strength of some combinatorial principles related to Ramsey's Theorem for Pairs, Vol. 15 of Lecture Notes Series, chapter 8, Institute of Mathematical Sciences, National University of Singapore (2008).

[5] J. H. Lutz, Gales and the constructive dimension of individual sequences, in Automata, languages and programming (Geneva, 2000), Vol. 1853 of Lecture Notes in Comput. Sci., 902-913, Springer, Berlin (2000).

[6] R. Lyons, Random walks and percolation on trees, Ann. Probab. 18 (1990), no. 3, 931-958.

[7] J. S. Miller, Extracting information is hard, Adv. Math. To appear.

[8] P. Mörters and Y. Peres, Brownian Motion. Draft available at http://www.stat.berkeley.edu/ peres/.

[9] J. Reimann, Effectively closed sets of measures and randomness, Ann. Pure Appl. Logic, 156 (2008), no. $1,170-182$.

[10] J. Reimann and F. Stephan, Effective Hausdorff dimension, in Logic Colloquium '01, Vol. 20 of Lect. Notes Log., 369-385, Assoc. Symbol. Logic, Urbana, IL (2005).

[11] S. G. Simpson, Subsystems of second order arithmetic, Perspectives in Mathematical Logic, Springer-Verlag, Berlin (1999), ISBN 3-540-64882-8.

Department of Mathematics, University of Hawai'i at MĀnoa, 2565 McCarthy Mall, HONOLULU HI 96822

E-mail address: bjoern@math.hawaii.edu

URL: http://www.math.hawaii.edu/ pjoern 